\title{
Depression, anxiety, and associated factors in patients with diabetes: evidence from the anxiety, depression, and personality traits in diabetes mellitus (ADAPT-DM) study
}

Luke Sy-Cherng Woon ${ }^{1}$, Hatta Bin Sidi ${ }^{1}$, Arun Ravindran², Paula Junggar Gosse², Roslyn Laurie Mainland², Emily Samantha Kaunismaa ${ }^{2}$, Nurul Hazwani Hatta ${ }^{1}$, Puteri Arnawati ${ }^{1}$, Amelia Yasmin Zulkifli ${ }^{1}$, Norlaila Mustafa ${ }^{3}$ and Mohammad Farris Iman Leong Bin Abdullah ${ }^{4^{*}}$ (i)

\begin{abstract}
Background: Depression and anxiety are common psychiatric complications affecting patients with diabetes mellitus. However, data on the prevalence of depression, anxiety, and associated factors among Malaysian diabetic patients is scarce. The Anxiety, Depression, and Personality Traits in Diabetes Mellitus (ADAPT-DM) study aimed to determine the prevalence of depression and anxiety, and their associated factors in the Malaysian diabetic population.

Methods: This cross-sectional study recruited 300 diabetic patients via convenience sampling from the Endocrine outpatient clinic of Universiti Kebangsaan Malaysia Medical Centre, a tertiary referral healthcare facility in Kuala Lumpur. Socio-demographic characteristics and clinical history were obtained from each participant. The Generalised Anxiety Disorder-7 (GAD-7) was administered to assess anxiety symptoms, the Beck Depression Inventory (BDI) to assess depressive symptoms, the Big Five Inventory (BFI) to evaluate personality traits, and the World Health Organization Quality of Life-BREF (WHOQOL-BREF) to measure quality of life (QOL). Stepwise multiple logistic regression analyses were performed to determine the association between various factors, and depression and anxiety.

(Continued on next page)
\end{abstract}

\footnotetext{
* Correspondence: farris@usm.my

${ }^{4}$ Lifestyle Science Cluster, Advanced Medical and Dental Institute, Universiti Sains Malaysia, SAINS@BERTAM, 13200 Kepala Batas, Pulau Pinang, Malaysia

Full list of author information is available at the end of the article
}

(c) The Author(s). 2020 Open Access This article is licensed under a Creative Commons Attribution 4.0 International License, which permits use, sharing, adaptation, distribution and reproduction in any medium or format, as long as you give appropriate credit to the original author(s) and the source, provide a link to the Creative Commons licence, and indicate if changes were made. The images or other third party material in this article are included in the article's Creative Commons licence, unless indicated otherwise in a credit line to the material. If material is not included in the article's Creative Commons licence and your intended use is not permitted by statutory regulation or exceeds the permitted use, you will need to obtain permission directly from the copyright holder. To view a copy of this licence, visit http://creativecommons.org/licenses/by/4.0/. The Creative Commons Public Domain Dedication waiver (http://creativecommons.org/publicdomain/zero/1.0/) applies to the data made available in this article, unless otherwise stated in a credit line to the data. 


\begin{abstract}
(Continued from previous page)
Results: The prevalence of depression was 20\% ( $n=60)$ while anxiety was 9\% $(n=27)$. Co-morbid depression (adjusted odds ratio $[\mathrm{OR}]=9.89,95 \%$ confidence interval $[\mathrm{Cl}]=2.63-37.14, p=0.001$ ) and neuroticism (adjusted $\mathrm{OR}=11.66,95 \% \mathrm{Cl}=2.69-50.47, p=0.001)$ increased the odds of developing anxiety, while conscientiousness (adjusted $\mathrm{OR}=0.45,95 \% \mathrm{Cl}=0.23-0.80, p=0.004$ ) and greater psychological-related $\mathrm{QOL}$ (adjusted $\mathrm{OR}=0.47,95 \%$ $\mathrm{Cl}=0.29-0.75, p=0.002$ ) were protective. Co-morbid anxiety (adjusted $\mathrm{OR}=19.83,95 \% \mathrm{Cl}=5.63-69.92, p<0.001$ ) increased the odds of depression, while older age (adjusted $\mathrm{OR}=0.96,95 \% \mathrm{Cl}=0.93-0.98, p=0.002$ ), social relationship-related $\mathrm{QOL}$ (adjusted $\mathrm{OR}=0.84,95 \% \mathrm{Cl}=0.71-.0 .99, p=0.047$ ), and physical health-related $\mathrm{QOL}$ (adjusted $\mathrm{OR}=0.69,95 \% \mathrm{Cl}=0.58-0.83, p<0.001$ ) were protective.

Conclusions: The study findings signify the need to screen for co-morbid depression and anxiety, as well as personality traits and QOL, and to include psychosocial interventions when planning a multidisciplinary approach to managing diabetes.
\end{abstract}

Keywords: Anxiety, Depression, Diabetes mellitus, Personality traits, Quality of life

\section{Background}

Diabetes mellitus is a heterogeneous disorder that can result in severe morbidity with substantial emotional impact. The International Diabetes Federation (IDF) has estimated that approximately 425 million adults between the age of $2-79$ years were afflicted with diabetes in 2017 , and this number is projected to increase up to 629 million by 2045 [1]. Although diabetes is an international health crisis, its prevalence is increasing more rapidly in lower- and middle-income countries [2].

In recent decades, research has focused on the mental health comorbidities associated with diabetes. The occurrence of anxiety and depressive disorders can be as high as two-fold greater in individuals suffering from diabetes. These mood disorders are linked to suboptimal diabetic self-care, unhealthy behaviours, elevated HbA1c, and other sub-optimal metabolic indicators [3-7].

Evidence suggests a bidirectional relationship between diabetes mellitus, and anxiety and depressive disorders. Patients with anxiety symptoms may be at increased risk of developing type 2 diabetes and vice versa [7]. Various factors may contribute to the development of anxiety disorders among patients with diabetes, including personal and family history, stressful life events, substance use, and physical illness [7]. It is possible that diabetes and depression stem from similar or shared aetiologies, or the existence of one condition may increase the prevalence of the other [8]. Possible risk factors that may contribute to the development of depression in diabetic patients are personal and family history, stressful life events, domestic violence, physical illnesses, and clinical factors [8-11].

The relationship between diabetes and psychiatric disorders is still not fully understood.

Personality traits and quality of life (QOL) may contribute to the development and severity of psychiatric disorders in patients with diabetes, but this has not been comprehensively studied. Furthermore, data on the prevalence of depression and anxiety among Malaysian diabetic patients are scarce. In fact, much of the research on diabetes as it relates to personality and mood disorders has been conducted in higher-income countries, despite the strong need to understand these relationships in lower- and middle-income countries where individuals may face additional barriers to care [12]. Hence, the Anxiety, Depression, and Personality Traits in Diabetes Mellitus (ADAPT-DM) study was conducted to examine the prevalence of depression and anxiety among Malaysian diabetic patients, and to investigate their associated socio-demographic characteristics, personality traits, and QOL.

\section{Methods \\ Study design and participants}

The ADAPT-DM study used a cross-sectional design and participants were recruited over a period of 2 months at the Endocrine outpatient clinic of Universiti Kebangsaan Malaysia Medical Centre (UKMMC), a tertiary referral centre in Kuala Lumpur, Malaysia. Approval was obtained from the Human Ethics Committee of the Faculty of Medicine, Universiti Kebangsaan Malaysia (UKM FPR.SPI 800-2/28/166/FF-2019-342). Sample size was calculated based on previous estimates of the prevalence of depression and generalized anxiety disorder in patients with diabetes, which are 20 and $14 \%$ respectively [13, 14]. The sample size required was 234 subjects. Study participants were recruited via convenience sampling, in which those who attended the Endocrine clinic as outpatients and interested in.

participating were provided with detailed explanations of the study by the researchers. Then, they were screened for inclusion criteria, such as (1) being 18 years and above and (2) having a confirmed diagnosis of type 1 , type 2, or gestational diabetes mellitus. Patients with impaired mental capacity, such as those with psychotic features or cognitive impairment, were excluded from 
the study. The eligible participants were allowed to participate in the study if verbal and written informed consent was given. All participants who were found to have depression and anxiety disorders were referred to Department of Psychiatry, UKMMC for further assessment.

\section{Measuring tools}

The participants completed a questionnaire which collected data on demographic, social, and clinical characteristics. The demographic variables included age, gender, marital status, ethnicity, education level, employment status, household income, and religion. The social variables included perceived level of social support, smoking, alcohol use, and recreational drug use. Clinical variables included medical history, diabetes history (onset, type, and use of insulin therapy), body mass index (BMI), and self-perceived management of diabetes (assessed using a five-point Likert scale). Information provided from the questionnaire was supplemented by a review of patient medical records where applicable. In addition, the participants were administered the sevenitem Generalised Anxiety Disorder scale (GAD-7) to assess the prevalence of anxiety, Beck Depression Inventory-II (BDI-II) to assess the prevalence of depression, Big Five Inventory (BFI) to assess personality traits, and the World Health Organization Quality of LifeBREF (WHOQOL-BREF) to measure quality of life (QOL).

\section{Seven-item generalised anxiety disorder scale (GAD-7)}

The GAD-7 is a self-reported questionnaire designed to screen for generalized anxiety disorder (GAD). It consists of seven items, with each item being assessed using a Likert scale of 0 to 3 . Hence, its total score ranges from 0 to 21. The GAD-7 has been found to be a good case-finding instrument for GAD. Participants with scores $\geq 8$ are classified as having GAD. The GAD-7 has proven to be reliable, with a sensitivity of $92 \%$ and specificity of $76 \%$ at cut-off point of $\geq 8$ [15]. The Malay version of the GAD-7 used in this study has been proven to be reliable, with a sensitivity of $76 \%$ and specificity of 94\% [16].

\section{Beck depression inventory-II (BDI-II)}

The BDI-II is a self-reported questionnaire commonly used to screen for and assess the severity of depression. It is comprised of items that are related to depressive symptoms. It is made up of 21 items and each scored from 0 to 3 . A score of 10 to 16 indicates mild depression, a score of 17 to 29 indicates moderate depression, and a score between 30 and 63 indicates severe depression [17]. The BDI-II has been proven to have good internal consistency, with Cronbach's $\alpha$ of 0.91. The Malay version of the BDI-II has also been deemed to have good internal consistency (Cronbach's $\alpha=0.71$ to 0.91 ) and excellent psychometric properties [18].

\section{Big five inventory (BFI)}

The BFI is a short instrument that assesses personality traits based on the Five Factor model. The BFI includes 44 items divided into five subscales: extraversion, agreeableness, conscientiousness, neuroticism, and openness. Each item is scored on a five-point Likert scale ranging from 0 (strongly agree) to 4 (strongly disagree) [19]. The Malay version of the BFI has been proven to have good internal consistency, as well as good convergent and discriminant validity [20]. There is no study which investigate the norms of the BFI subscale scores in the general population. Nevertheless, a study with a large sample of American adults aged 21 to 60 years $(n=132,515)$ indicated that the range of the mean of the BFI subscale scores were as follow: $3.10(\mathrm{SD}=0.85)$ to 3.31 ( $\mathrm{SD}=$ $0.90)$ (extraversion), 3.64 $(\mathrm{SD}=0.72)$ to $4.01(\mathrm{SD}=0.67)$ (agreeableness), $3.45(\mathrm{SD}=0.73)$ to $3.93 \quad(\mathrm{SD}=0.73)$ (conscientiousness), $2.92(\mathrm{SD}=0.99)$ to $3.32(\mathrm{SD}=0.82)$ (neuroticism), and $3.79(\mathrm{SD}=0.73)$ to $3.96(\mathrm{SD}=0.66)$ (openness) [21].

\section{World Health Organization quality of life-BREF (WHOQOL- BREF)}

The WHOQOL-BREF is a self-reported questionnaire that assesses quality of life. It is comprised of 26 items. Items 1 and 2 assess overall quality of life, while the remaining items are grouped into four categories that evaluate different domains: physical health, psychological, social relationships, and environmental quality of life. Each item is scored on a Likert scale ranging from 1 to 5. The WHOQOL-BREF has good psychometric properties and has been proven to be a valid and reliable alternative to the WHOQOL-100 for measuring quality of life [22]. The Malay version of the WHOQOL-BREF has also demonstrated excellent psychometric properties with internal consistency (Cronbach's $\alpha$ ) of 0.89 [23]. The general norms for the WHOQOL-BREF domains were $73.5(\mathrm{SD}=18.1)$ for physical health quality of life, $70.6(\mathrm{SD}=14.0)$ for psychological quality of life, 71.5 $(\mathrm{SD}=18.2)$ for social relationships quality of life, and $75.1(\mathrm{SD}=13.0)$ for environmental quality of life [24].

\section{Data analysis}

Statistical analysis was carried out using the Statistical Package for Social Science (SPSS) (version 20.0; IBM, Armonk, NY). Descriptive statistics were computed in which categorical variables were reported in frequency and percentage, and continuous variables reported in median and interquartile range (IQR). The continuous variables were not normally distributed as demonstrated by the Kolmogorov-Smirnov test $(p<0.05)$. The missing 
data was resolved with mode imputation as the variables with missing data were all categorical variables. Initially, univariate logistic regression was performed to detect any individual association between demographic, social, and clinical characteristics, the BFI and the WHOQOLBREF scores (independent variables) and anxiety and depression $(0=$ absence of anxiety/depression, $1=$ presence of anxiety/depression) (dependent variable). Variables with $p<0.25$ were entered into a stepwise multiple logistic regression model as independent variables to determine the factors which significantly predict occurrence of anxiety and depression among participants. The goodness-of-fit of the stepwise multiple logistic regression model was assessed with the HosmerLemeshow goodness-of-fit test, in which $p>0.05$ was considered as good model fit. The ability of the model to discriminate participants with presence and absence of anxiety/depression was assessed with the area under the receiver operating characteristic (ROC) curve. The area under the curve (AUC) of $>0.7$ was considered as acceptable fit. Statistical significance for all analyses was set to $p<0.05$.

\section{Results}

Demographic, social, and clinical characteristics, personality traits, and quality of life

The demographic, social, and clinical characteristics of participants, as well as their personality traits and quality of life, are summarized in Table 1 . A total of 300 participants were enrolled in the study. The median age of the participants was 63 years $(\mathrm{IQR}=16$ years). Approximately half of the participants were males $(n=158,52.7 \%)$. More than half of the participants were Malays $(n=195,65.0 \%)$ and a similar proportion of participants were Muslim $(n=199$, $66.3 \%)$. Large proportion of the participants also agreed that they had regular religious practice $(n=$ $237,79.0 \%)$. The majority of participants were married $(n=233,77.7 \%)$, and an almost equal proportion of participants received up to a secondary level of education $(n=133,44.3 \%)$ or a tertiary level of education $(n=119,39.7 \%)$. A minority of participants were retired $(n=123,41 \%)$, and slightly more than half were earning less than RM 3000 per month $(n=166,55.3 \%)$. The majority of participants perceived that they had good social support, with $80.3 \%$ $(n=241)$ of participants 'agreeing' with adequate social support. The majority of participants had never smoked $(n=216,72.0 \%)$, had never consumed alcohol $(n=271,90.3 \%)$, and had no history of recreational drug use $(n=294,98 \%)$.

With regard to clinical characteristics, the majority of participants were diagnosed with type 2 diabetes mellitus $(n=269,89.7 \%)$, while a minority had been diagnosed with type 1 diabetes mellitus $(n=22,7.3 \%)$ or gestational diabetes mellitus $(n=6,2.0 \%)$. The median duration of diabetes diagnosis was 14 years $\left(\mathrm{IQR}=12\right.$ years), while the median $\mathrm{HbAl}_{\mathrm{C}}$ measurement was $7.6 \%(\mathrm{IQR}=2.7 \%)$. Almost half of the participants were on insulin therapy $(n=138,46.0 \%)$. More than half of the participants perceived that they managed their illness well; in fact, $71.3 \%(n=214)$ of participants 'agreed' with the statement 'I am able to manage my diabetes well'. Almost half $(49.0 \%, n=$ 147) of participants were overweight (BMI 25-30), while $26.0 \%(n=78)$ were obese (BMI $>30)$.

Screening with the GAD-7 indicated that only a small proportion of the participants had anxiety $(9 \%, n=27)$, while BDI-II screening revealed that a relatively larger proportion of participants had depression $(20 \%, n=60)$. In BFI assessment, the median of extraversion was 3.38 $(\mathrm{IQR}=0.75)$, agreeableness was $3.78(\mathrm{IQR}=0.43)$, conscientiousness was $3.67(\mathrm{IQR}=0.60)$, neuroticism was $2.50(\mathrm{IQR}=0.73)$, and openness was $3.30(\mathrm{IQR}=0.60)$. The WHOQOL-BREF screening revealed that the median of the physical health score was $14.29(\mathrm{IQR}=3.43)$, the psychological score was $15.33(\mathrm{IQR}=2.67)$, the social relationships score was $16.00(\mathrm{IQR}=2.67)$, and the environment score was $15.00(\mathrm{IQR}=2.50)$. The mean and standard deviation (SD) of the BFI subscale scores and the WHOQOL-BREF domain scores are summarized in Table $\mathrm{S} 1$ in Additional file 1 (supplementary information).

The association between demographic, social, and clinical characteristics, personality traits and quality of life, and anxiety among participants

The findings of the univariate logistic regression analyses examining associations between demographic, social, and clinical characteristics, personality traits, quality of life, and anxiety are summarized in Table 2 . The demographic characteristics associated with anxiety $(p<0.25)$ were ethnicity, employment status, household income, and regular religious practice. There were no significant association between history of cigarette smoking, alcohol intake, and recreational drug use, and anxiety. On the contrary, there were several clinical characteristics, personality traits, and quality of life components which were associated with anxiety. The variables which were associated with anxiety include self-perceived diabetic management, depression, overall perception of QOL, overall perception of health, physical quality of life, psychological quality of life, social quality of life, environmental quality of life, extraversion, agreeableness, conscientiousness, neuroticism, and openness scores, and the interaction between perceived social support and neuroticism. 
Table 1 Socio-demographic, social and clinical characteristics of the participants

\begin{tabular}{l} 
Variables \\
\hline Age $(N=300)$ \\
Gender \\
Male \\
Female \\
Missing \\
Ethnicity \\
Malay \\
Chinese \\
Indian \\
Others
\end{tabular}

Marital status

Married

Single

Divorced/widowed

Education

None
Primary
Secondary
Tertiary
Missing
Employment

Employment

Employed

Unemployed

Retired

Missing

Household income

$<$ RM3,000

RM3,000-6000

$>\mathrm{RM} 6,000$

Missing

Religion

Islam

Buddhism

Hindusim

Christianity

Others

Missing

Regular religious practice

Disagree
Neutral
Agree
Missing
Smoking

\begin{tabular}{ll}
$N$ & $\%$ \\
\hline $63^{\mathrm{a}}$ & $16^{\mathrm{b}}$
\end{tabular}

158

141

1

195

54

44

7

233

24

43

7

38

133

119

3

80

94

123

3

52.7

47.0

0.3

65.0

18.0

14.7

2.3

77.7

8.0

14.3

(4)

2.3

12.7

44.3

39.7

1.0

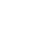

166

57

61

16

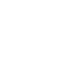

199

37

35

23

4

2

19

43

237

1

1

31.3

41.0

Table 1 Socio-demographic, social and clinical characteristics of the participants (Continued)

\begin{tabular}{lll}
\hline Variables & $\mathrm{N}$ & $\%$ \\
\hline Never & 216 & 72.0 \\
Ex-smoker & 64 & 21.3 \\
$\quad$ Current smoker & 20 & 6.7 \\
Alcohol use & & \\
Yes & 26 & 8.7 \\
No & 271 & 90.3 \\
Missing & 3 & 1.0 \\
Recreational drug use & & \\
Yes & 5 & 1.7 \\
No & 294 & 98.0 \\
Missing & 1 & 0.3
\end{tabular}

Perceived social support

Very poor
Poor
Neutral
Good
Very good
Missing

2

8

2.7

48

16.0

$165 \quad 55.0$

$76 \quad 25.3$

$1 \quad 0.3$

Diabetes type

$\begin{array}{lll}\text { Type } 1 & 22 & 7.3\end{array}$

Type 2

89.7

Gestational

269

2.0

Missing

Duration of DM (years) $(N=229)$

1.0

Insulin therapy

$\begin{array}{lll}\text { Yes } & 138 & 46.0 \\ \text { No } & 114 & 38.0 \\ \text { Missing } & 48 & 16.0 \\ \text { HbA1c (\%) }(N=268) & 7.6^{\mathrm{a}} & 2.7^{\mathrm{b}} \\ \text { Diabetic control } & & \\ \text { Good } & 92 & 30.7 \\ \text { Poor } & 208 & 69.3\end{array}$

12.3 "I am able to manage my diabetes well"

69.3

$\begin{array}{lll}11.7 & \text { Disagree } & 15\end{array}$

$\begin{array}{llll}7.7 & \text { Neutral } & 70 & 23.4\end{array}$

$\begin{array}{llll}7.3 & \text { Agree } & 214 & 71.3\end{array}$

$\begin{array}{llll}0.7 & \text { Missing } & 1 & 0.3\end{array}$

Obesity

$\begin{array}{llll}6.3 & \text { BMI }<25 & 65 & 21.7 \\ 14.4 & \text { BMI 25-30 } & 147 & 49.0 \\ 79.0 & \text { BMI }>30 & 78 & 26.0 \\ 0.3 & \text { Missing } & 10 & 3.3\end{array}$

Hypertension 
Table 1 Socio-demographic, social and clinical characteristics of the participants (Continued)

\begin{tabular}{|c|c|c|}
\hline Variables & $\mathrm{N}$ & $\%$ \\
\hline Yes & 222 & 74.0 \\
\hline No & 78 & 26.0 \\
\hline \multicolumn{3}{|l|}{ Dyslipidaemia } \\
\hline Yes & 152 & 50.7 \\
\hline No & 148 & 49.3 \\
\hline \multicolumn{3}{|l|}{ Ischaemic heart disease } \\
\hline Yes & 82 & 27.3 \\
\hline No & 218 & 72.7 \\
\hline \multicolumn{3}{|l|}{ Stroke } \\
\hline Yes & 27 & 9.0 \\
\hline No & 273 & 91.0 \\
\hline \multicolumn{3}{|l|}{ Renal disease } \\
\hline Yes & 53 & 17.7 \\
\hline No & 247 & 82.3 \\
\hline \multicolumn{3}{|l|}{ Anxiety (GAD-7) } \\
\hline Yes & 27 & 9.0 \\
\hline No & 273 & 91.0 \\
\hline \multicolumn{3}{|l|}{ Depression (BDI) } \\
\hline No/minimal & 240 & 80.0 \\
\hline Mild to moderate & 41 & 13.7 \\
\hline Moderate to severe & 17 & 5.7 \\
\hline Severe & 2 & 0.7 \\
\hline \multicolumn{3}{|l|}{ WHOQOL-BREF } \\
\hline \multicolumn{3}{|l|}{ Overall perception of QOL } \\
\hline Very poor & 3 & 1.0 \\
\hline Poor & 5 & 1.7 \\
\hline Neither poor nor good & 89 & 29.7 \\
\hline Good & 162 & 54.0 \\
\hline Very good & 41 & 13.7 \\
\hline \multicolumn{3}{|l|}{ Overall perception of health } \\
\hline Very dissatisfied & 1 & 0.3 \\
\hline Dissatisfied & 37 & 12.3 \\
\hline Neither satisfied nor dissatisfied & 131 & 43.7 \\
\hline Satisfied & 121 & 40.3 \\
\hline Very satisfied & 10 & 3.3 \\
\hline \multicolumn{3}{|l|}{ WHOQOL-BREF domains $(\mathrm{N}=300)$} \\
\hline Physical health & $63.00^{\mathrm{a}}$ & $19.00^{\mathrm{b}}$ \\
\hline Psychological & $69.00^{\mathrm{a}}$ & $13.53^{\mathrm{b}}$ \\
\hline Social relationships & $75.00^{\mathrm{a}}$ & $19.00^{\mathrm{b}}$ \\
\hline Environment & $69.00^{\mathrm{a}}$ & $12.00^{\mathrm{b}}$ \\
\hline \multicolumn{3}{|l|}{ BFI subscales $(N=300)$} \\
\hline Extraversion & $3.38^{\mathrm{a}}$ & $0.75^{\mathrm{b}}$ \\
\hline Agreeableness & $3.78^{\mathrm{a}}$ & $0.43^{\mathrm{b}}$ \\
\hline
\end{tabular}

Table 1 Socio-demographic, social and clinical characteristics of the participants (Continued)

\begin{tabular}{cll}
\hline Variables & $\mathrm{N}$ & $\%$ \\
\hline Conscienciousness & $3.67^{\mathrm{a}}$ & $0.60^{\mathrm{b}}$ \\
Neuroticism & $2.50^{\mathrm{a}}$ & $0.73^{\mathrm{b}}$ \\
Openness & $3.30^{\mathrm{a}}$ & $0.60^{\mathrm{b}}$ \\
\hline${ }^{\mathrm{a}}=$ Median; ${ }^{\mathrm{b}}=$ Interquartile range (IQR) & &
\end{tabular}

The association between demographic, social, and clinical characteristics, personality traits and quality of life, and depression among participants

The findings of the univariate logistic regression analyses examining the associations between demographic, social, and clinical characteristics, personality traits, quality of life, and depression among participants are summarized in Table 3. There were four demographic characteristics associated with depression $(p<0.25)$, such as age, employment status, household income, and regular religious practice. There were no significant associations between social characteristics and depression among participants. Several clinical characteristics, personality traits, and quality of life components were associated with depression. The variables which were associated with depression include self-perceived diabetic management, anxiety, overall perception of QOL, overall perception of health, physical quality of life, psychological quality of life, social quality of life, environmental quality of life, extraversion, agreeableness, conscientiousness, and neuroticism scores, and the interaction between perceived social support and neuroticism.

\section{Stepwise multiple logistic regression analyses between various factors and anxiety among participants}

The findings of stepwise multiple logistic regression analyses between demographic characteristics (ethnicity, employment, household income, and practice of religion), clinical factors (co-morbid depression), personality traits (extraversion, agreeableness, neuroticism, conscientiousness, openness, and the interaction between perceived social support and neuroticism), quality of life (overall perception of QOL, overall perception of health, physical, psychological, social, and environmental QOL), and anxiety among participants are summarized in Table 4. There were only a few factors predictive of anxiety among participants. Participants who were depressed (adjusted OR $=9.89,95 \% \mathrm{CI}=2.63-37.14, p=$ 0.001 ) with higher neuroticism scores (adjusted $\mathrm{OR}=$ 11.66, 95\% CI $=2.69-50.47, p=0.001$ ) had higher odds of having anxiety. On the contrary, lower odds of anxiety was associated with higher psychological scores on the quality of life questionnaire (adjusted OR $=0.47,95 \%$ $\mathrm{CI}=0.29-0.75, p=0.002)$ and higher conscientiousness scores (adjusted $\mathrm{OR}=0.45,95 \% \mathrm{CI}=0.23-0.80, p=$ 
Table 2 The association between individual socio-demographic, social and clinical characteristics, and anxiety among participants

\begin{tabular}{|c|c|c|}
\hline Variables & Crude OR $(95 \% \mathrm{Cl})$ & $p$-value \\
\hline Age & $0.99(0.96-1.02)$ & 0.585 \\
\hline \multicolumn{3}{|l|}{ Gender } \\
\hline Male & 1 & \\
\hline Female & $0.75(0.34-1.68)$ & 0.485 \\
\hline \multicolumn{3}{|l|}{ Ethnicity: } \\
\hline Non-Malays & 1 & \\
\hline Malays & $0.39(0.18-0.88)$ & $0.022^{*}$ \\
\hline \multicolumn{3}{|l|}{ Marital status: } \\
\hline Married & 1 & \\
\hline Not married & $1.73(0.58-5.17)$ & 0.331 \\
\hline \multicolumn{3}{|l|}{ Education: } \\
\hline Secondary \& below & 1 & \\
\hline Tertiary & $1.05(0.47-2.35)$ & 0.905 \\
\hline \multicolumn{3}{|l|}{ Employment: } \\
\hline Employed & 1 & \\
\hline Unemployed & $0.53(0.20-1.36)$ & $0.186^{*}$ \\
\hline Retired & $0.34(0.13-0.91)$ & $0.032^{*}$ \\
\hline \multicolumn{3}{|l|}{ Household income: } \\
\hline$<\mathrm{RM} 3,000$ & 1 & \\
\hline RM3,000-6000 & $0.41(0.12-1.42)$ & $0.158^{*}$ \\
\hline$>\mathrm{RM} 6,000$ & $0.38(0.11-1.32)$ & $0.127^{*}$ \\
\hline \multicolumn{3}{|l|}{ Regular religious practice: } \\
\hline Disagree & 1 & \\
\hline Neutral & $0.18(0.03-1.06)$ & $0.058^{*}$ \\
\hline Agree & $0.34(0.10-1.11)$ & $0.074^{*}$ \\
\hline \multicolumn{3}{|l|}{ Perceived social support: } \\
\hline Poor & 1 & \\
\hline Neutral & $1.05(0.19-5.76)$ & 0.953 \\
\hline Good & $0.26(0.05-1.36)$ & 0.311 \\
\hline \multicolumn{3}{|l|}{ Cigarette smoking: } \\
\hline Non-smoker & 1 & \\
\hline Smoker & $1.58(0.69-3.61)$ & 0.276 \\
\hline \multicolumn{3}{|l|}{ Alcohol: } \\
\hline No & 1 & \\
\hline Yes & $0.38(0.05-2.93)$ & 0.354 \\
\hline \multicolumn{3}{|l|}{ Recreational drug use: } \\
\hline No & 1 & \\
\hline Yes & $2.59(0.28-24.01)$ & 0.403 \\
\hline \multicolumn{3}{|l|}{ Obesity: } \\
\hline $\mathrm{BMI}<25$ & 1 & \\
\hline BMI 25-30 & $1.99(0.64-6.14)$ & 0.254 \\
\hline $\mathrm{BMI}>30$ & $1.33(0.36-4.94)$ & 0.667 \\
\hline \multicolumn{3}{|l|}{ Diabetes mellitus type: } \\
\hline Type I or gestational diabetes & 1 & \\
\hline
\end{tabular}


Table 2 The association between individual socio-demographic, social and clinical characteristics, and anxiety among participants (Continued)

\begin{tabular}{|c|c|c|}
\hline Variables & Crude OR (95\% CI) & $p$-value \\
\hline Type II diabetes & $2.74(0.36-21.00)$ & 0.333 \\
\hline \multicolumn{3}{|l|}{ Insulin therapy: } \\
\hline No & 1 & \\
\hline Yes & $1.51(0.64-3.57)$ & 0.350 \\
\hline \multicolumn{3}{|l|}{ Good self-perceived diabetic management: } \\
\hline Disagree & 1 & \\
\hline Neutral & $0.19(0.05-0.69)$ & $0.011^{*}$ \\
\hline Agree & $0.10(0.03-0.31)$ & $<0.001^{*}$ \\
\hline \multicolumn{3}{|l|}{ Diabetic control: } \\
\hline Good & 1 & \\
\hline Poor & $1.61(0.63-4.13)$ & 0.322 \\
\hline \multicolumn{3}{|l|}{ Depression: } \\
\hline No depression & 1 & \\
\hline Depression & $36.68(12.00-112.06)$ & $<0.001^{*}$ \\
\hline \multicolumn{3}{|l|}{ Overall perception of QOL: } \\
\hline Poor/very poor & 1 & \\
\hline Neutral & $0.10(0.02-0.48)$ & $0.004^{*}$ \\
\hline Good/very good & $0.03(0.01-0.14)$ & $<0.001^{*}$ \\
\hline \multicolumn{3}{|l|}{ Overall perception of health } \\
\hline Poor/very poor & 1 & \\
\hline Neutral & $0.18(0.07-0.46)$ & $<0.001^{*}$ \\
\hline Good/very good & $0.09(0.03-0.27)$ & $<0.001^{*}$ \\
\hline \multicolumn{3}{|l|}{ WHOQOL-BREF domains } \\
\hline Physical health & $0.72(0.61-0.85)$ & $<0.001^{*}$ \\
\hline Psychological & $0.48(0.38-0.62)$ & $<0.001^{*}$ \\
\hline Social relationships & $0.61(0.51-0.74)$ & $<0.001^{*}$ \\
\hline Environment & $0.60(0.48-0.56)$ & $<0.001^{*}$ \\
\hline \multicolumn{3}{|l|}{ BFI subscales } \\
\hline Extraversion & $0.38(0.17-0.85)$ & $0.018^{*}$ \\
\hline Agreeableness & $0.20(0.07-0.54)$ & $0.001^{*}$ \\
\hline Conscientiousness & $0.52(0.22-1.23)$ & $0.135^{*}$ \\
\hline Neuroticism & $16.84(6.16-40.01)$ & $<0.001^{*}$ \\
\hline Openness & $1.91(0.85-4.29)$ & $0.119^{*}$ \\
\hline Neuroticism x perceived social support & $1.24(1.06-1.46)$ & $0.009^{*}$ \\
\hline
\end{tabular}

${ }^{*} p$-value $<0.25$

0.004). Other demographic characteristics, personality traits, and QOL components were not significant predictors of occurrence of anxiety among the participants. The logistic regression model reported a Cox and Snell $\mathrm{R}^{2}$ of 0.29 $(p<0.001)$, Hosmer-Lemeshow goodness-of-fit test was not significant $(p=0.843)$ and the area under the ROC curve (AUC) of 0.949 (95\% CI =0.912-0.986, p < 0.001), indicating acceptable fit of the model to discriminate participants with presence and absence of anxiety.
Stepwise multivariate logistic regression analyses between various factors and depression among participants

The findings of stepwise multivariate logistic regression analyses between demographic characteristics (age, employment, household income, and practice of religion), clinical factors (co-morbid anxiety and perceived diabetic management), personality traits (extraversion, agreeableness, conscientiousness, neuroticism, and the 
Table 3 The association between individual socio-demographic, social and clinical characteristics, and depression among participants

\begin{tabular}{|c|c|c|}
\hline Variables & Crude OR (95\% CI) & $p$-value \\
\hline Age & $0.98(0.96-0.99)$ & $0.030^{*}$ \\
\hline \multicolumn{3}{|l|}{ Gender } \\
\hline Male & 1 & \\
\hline Female & $0.76(0.43-1.34)$ & 0.341 \\
\hline \multicolumn{3}{|l|}{ Ethnicity } \\
\hline Non-Malays & 1 & \\
\hline Malays & $0.84(0.47-1.50)$ & 0.545 \\
\hline \multicolumn{3}{|l|}{ Marital status: } \\
\hline Married & 1 & \\
\hline Not married & $1.05(0.53-2.08)$ & 0.890 \\
\hline \multicolumn{3}{|l|}{ Education } \\
\hline Secondary \& below & 1 & \\
\hline Tertiary & $0.85(0.48-1.53)$ & 0.596 \\
\hline \multicolumn{3}{|l|}{ Employment } \\
\hline Employed & 1 & \\
\hline Unemployed & $0.91(0.46-1.81)$ & 0.788 \\
\hline Retired & $0.42(0.20-0.87)$ & $0.019^{*}$ \\
\hline \multicolumn{3}{|l|}{ Household income } \\
\hline$<\mathrm{RM} 3,000$ & 1 & \\
\hline $\mathrm{RM} 3,000-6000$ & $0.96(0.41-1.97)$ & 0.916 \\
\hline$>\mathrm{RM} 6,000$ & $0.36(0.14-0.89)$ & $0.027^{*}$ \\
\hline \multicolumn{3}{|l|}{ Regular religious practice } \\
\hline Disagree & 1 & \\
\hline Neutral & $0.24(0.07-0.78)$ & $0.018^{*}$ \\
\hline Agree & $0.22(0.08-0.58)$ & $0.002^{*}$ \\
\hline \multicolumn{3}{|l|}{ Perceived social support } \\
\hline Poor & 1 & \\
\hline Neutral & $2.40(0.46-12.57)$ & 0.300 \\
\hline Good & $0.79(0.16-3.87)$ & 0.773 \\
\hline \multicolumn{3}{|l|}{ Cigarette smoking } \\
\hline Non-smoker & 1 & \\
\hline Smoker & $1.13(0.61-2.10)$ & 0.700 \\
\hline \multicolumn{3}{|l|}{ Alcohol } \\
\hline No & 1 & \\
\hline Yes & $0.50(0.14-1.71)$ & 0.268 \\
\hline \multicolumn{3}{|l|}{ Recreational drug use } \\
\hline No & 1 & \\
\hline Yes & $0.98(0.11-9.10)$ & 0.988 \\
\hline \multicolumn{3}{|l|}{ Obesity } \\
\hline $\mathrm{BMI}<25$ & 1 & \\
\hline BMI 25-30 & $1.32(0.64-2.75)$ & 0.454 \\
\hline $\mathrm{BMI}>30$ & $1.02(0.44-2.39)$ & 0.962 \\
\hline \multicolumn{3}{|l|}{ Diabetes mellitus type } \\
\hline Type I or gestational diabetes & 1 & \\
\hline
\end{tabular}


Table 3 The association between individual socio-demographic, social and clinical characteristics, and depression among participants (Continued)

\begin{tabular}{|c|c|c|}
\hline Variables & Crude OR (95\% Cl) & $p$-value \\
\hline Type II diabetes & $1.11(0.40-3.06)$ & 0.840 \\
\hline \multicolumn{3}{|l|}{ Insulin therapy: } \\
\hline No & 1 & \\
\hline Yes & $0.83(0.46-1.47)$ & 0.513 \\
\hline \multicolumn{3}{|l|}{ Good self-perceived diabetic management } \\
\hline Disagree & 1 & \\
\hline Neutral & $0.49(0.16-1.53)$ & $0.218^{*}$ \\
\hline Agree & $0.20(0.07-0.59)$ & $0.004^{*}$ \\
\hline \multicolumn{3}{|l|}{ Diabetic control } \\
\hline Good & 1 & \\
\hline Poor & $1.42(0.74-2.70)$ & 0.289 \\
\hline \multicolumn{3}{|l|}{ Anxiety } \\
\hline No Anxiety & 1 & \\
\hline Anxiety & $36.68(12.00-112.06)$ & $<0.001^{*}$ \\
\hline \multicolumn{3}{|l|}{ Overall perception of QOL } \\
\hline Poor/very poor & 1 & \\
\hline Neutral & $0.14(0.03-0.73)$ & $0.019^{*}$ \\
\hline Good/very good & $0.05(0.01-0.28)$ & $<0.001^{*}$ \\
\hline \multicolumn{3}{|l|}{ Overall perception of health } \\
\hline Poor/very poor & 1 & \\
\hline Neutral & $0.46(0.21-0.98)$ & $<0.045^{*}$ \\
\hline Good/very good & $0.20(0.09-0.46)$ & $<0.001^{*}$ \\
\hline \multicolumn{3}{|l|}{ WHOQOL-BREF domains } \\
\hline Physical health & $0.66(0.58-0.76)$ & $<0.001^{*}$ \\
\hline Psychological & $0.58(0.49-0.61)$ & $<0.001^{*}$ \\
\hline Social relationships & $0.68(0.59-0.77)$ & $<0.001^{*}$ \\
\hline Environment & $0.66(0.56-0.78)$ & $<0.001^{*}$ \\
\hline \multicolumn{3}{|l|}{ BFI subscales } \\
\hline Extraversion & $0.50(0.28-0.89)$ & $0.018^{*}$ \\
\hline Agreeableness & $0.36(0.18-0.73)$ & $0.004^{*}$ \\
\hline Conscientiousness & $0.33(0.17-0.63)$ & $0.001^{*}$ \\
\hline Neuroticism & $5.54(3.02-10.18)$ & $<0.001^{*}$ \\
\hline Openness & $1.23(0.70-2.17)$ & 0.476 \\
\hline Neuroticism x perceived social support & $1.19(1.06-1.33)$ & $0.004^{*}$ \\
\hline
\end{tabular}

* $p$-value $<0.25$

interaction between perceived social support and neuroticism), quality of life (overall perception of QOL and health, physical, psychological, social, and environmental QOL), and depression among participants are summarized in Table 5 . The only clinical factor associated with higher odds of depression was anxiety, which increased the occurrence of depression by almost 20-fold (adjusted $\mathrm{OR}=19.83,95 \% \mathrm{CI}=5.63-69.92, p<0.001)$. On the contrary, older age (adjusted OR $=0.96,95 \% \mathrm{CI}=0.93-$
0.98, $p=0.002$ ), higher physical health quality of life scores (adjusted $\mathrm{OR}=0.69, \quad 95 \% \mathrm{CI}=0.58-0.83$, $p<0.001$ ), and higher social quality of life scores (adjusted $\mathrm{OR}=0.84,95 \% \mathrm{CI}=0.71-.0 .99, p=0.047$ ) were associated with lower odds of occurrence of depression. Perceived diabetic management, other demographic characteristics, personality traits, and QOL components did not significantly predict depression among the participants. The logistic regression model reported a Cox 
Table 4 Stepwise multiple logistic regression model between various factors and anxiety among participants

\begin{tabular}{lll}
\hline Variables & Adjusted $\mathrm{OR}^{\mathrm{a}}(95 \% \mathrm{Cl})$ & $p$-value \\
\hline Depression & & \\
No depression & 1 & $9.89(2.63-37.14)$ \\
Depression & $0.47(0.29-0.75)$ & $0.001^{*}$ \\
Psychological domain of WHOQOL-BREF & $0.45(0.23-0.80)$ & $0.002^{*}$ \\
Conscientiousness & $11.66(2.69-50.47)$ & $0.004^{*}$ \\
Neuroticism & $0.001^{*}$ \\
\hline
\end{tabular}

*Statistical significance at $p<0.05,{ }^{a}$ The stepwise logistic regression model indicated ethnicity, employment, household income, practice of religion, perceived diabetic management, overall perception of QOL, overall perception of health, physical, social relationship and environment domains of QOL, extraversion, agreeableness, openness personality traits, and neuroticism $\mathrm{x}$ perceived social support were not significantly associated with anxiety among the participants. The model reported reported Cox and Snell $\mathrm{R}^{2}=0.29, p<0.001$, Hosmer-Lemeshow goodness-of-fit test $(p=0.843)$, and the area under the receiver operating characteristic curve $(\mathrm{AUC})=0.949(95 \% \mathrm{Cl}=0.912-0.986, p<0.001)$

and Snell $\mathrm{R}^{2}$ of $0.294(p<0.001)$, Hosmer-Lemeshow goodness-of-fit test was not significant $(p=0.447)$ and the area under the ROC curve (AUC) of 0.851 (95\% CI = $0.793-0.909, p<0.001$ ), indicating acceptable fit of the model to discriminate participants with presence and absence of depression.

\section{Discussion}

The ADAPT-DM study aimed to determine the prevalence of depression and anxiety, and their associated factors among Malaysian patients with diabetes. Regarding the prevalence of depression and anxiety, we found that 9\% of the participants screened positive for anxiety and $20 \%$ met criteria for depression. The prevalence of depression reported in our study was similar to that reported in previous studies, where prevalence was estimated between 18 and 30\% [25]. The prevalence of anxiety among participants in our study was relatively low compared to the prevalence reported by the INTERPRET-DD study, which estimated the prevalence

Table 5 Stepwise multiple logistic regression model between various factors and depression among participants

\begin{tabular}{lll}
\hline Variables & Adjusted $\mathrm{OR}^{\mathrm{a}}(95 \% \mathrm{Cl})$ & $p$-value \\
\hline Age & $0.96(0.93-0.98)$ & $0.002^{*}$ \\
Anxiety: & & \\
$\quad$ No & 1 & \\
Yes & $19.83(5.63-69.92)$ & $<0.001^{*}$ \\
$\quad \begin{array}{l}\text { Physical domain of WHOQOL-BREF } \\
\text { Social relationship domain of WHOQOL- }\end{array}$ & $0.69(0.58-0.83)$ & $<0.001^{*}$ \\
$\quad$ BREF & $0.84(0.71-.0 .99)$ & $0.047^{*}$ \\
\hline
\end{tabular}

*Statistical significance at $p<0.05,{ }^{\text {a }}$ The stepwise logistic regression model indicated employment, household income, practice of religion, perceived diabetic management, overall perception of QOL, overall perception of health, psychological and environment domains of QOL, extraversion, agreeableness, conscientiousness, neuroticism personality traits, and neuroticism $\mathrm{x}$ perceived social support were not significantly associated with depression among the participants. The model reported reported Cox and Snell $R^{2}=0.294, p<0.001$, Hosmer-Lemeshow goodness-of-fit test $(p=0.447)$, and the area under the receiver operating characteristic curve (AUC) $=0.851(95 \%$

$\mathrm{Cl}=0.793-0.909, p<0.001)$ of anxiety (all anxiety disorder included) to be $18 \%$ based on data collected from 3170 diabetic patients from 15 countries in different continents [26]. This may be explained by the difference in instruments used for assessing anxiety. While we used the GAD-7, which is designed to assess for generalized anxiety disorder (GAD), the INTERPRET-DD study used the Mini International Neuropsychiatric Interview. The prevalence of GAD specifically reported by the INTERPRET-DD study was $8.1 \%$, which was similar to the prevalence of anxiety disorder reported in our study [26].

Our findings reveal that neuroticism and depression increased the odds of developing anxiety by almost 12 fold and 10-fold respectively. Better psychological QOL and higher conscientiousness were protective against anxiety which reduced the occurrence of anxiety by half (0.47-fold and 0.45 -fold respectively). The occurrence of depression greatly increased the odds of anxiety in our study, which is similar to what was reported in a study of 893 Chinese patients with diabetes [27]. The positive correlation between depression and anxiety is well documented in chronic illness, and the occurrence of depression can increase the risk of anxiety symptoms in patients with chronic illness [28, 29]. This relationship is expected as some theories suggested that anxiety and depression shared the same neurobiological mechanism in which they represent different phenotypic manifestations which run in a continuum [30]. The association between neuroticism and anxiety disorders, particularly generalized anxiety disorder and panic disorder, is well documented in the general population [31]. People with trait neuroticism tend to utilize maladaptive forms of emotional regulation rather than reappraisal which is believed to increase the severity of anxiety symptoms in these individuals [32]. However, higher psychological QOL reduced the odds of anxiety disorders in diabetic patients, which is in line with the findings of other studies on patients with diabetes [33, 34]. As expected, conscientiousness is inversely related to anxiety disorders, such as generalized anxiety disorder, panic disorder, 
agoraphobia, and social phobia [35]. A person with high conscientiousness possess traits, such as orderliness, high self-efficacy, achievement-driven, self-discipline, and responsible. Hence, a person with high conscientiousness tend to find stressful situations to be less demanding as they utilise adaptive coping strategies to overcome the effects of stressful situations, such as effective cognitive restructuring and instrumental problem solving. As a result, these traits in a person with high conscientiousness may help to reduce anxiety [32].

Our study indicated that among demographic and social characteristics, older age reduced odds of depression by 0.96 -fold. Regarding clinical characteristics, those with anxiety had almost 20-fold increased odds of developing depression. Greater physical health-related QOL and higher social relationship-related QOL reduced the occurrence of depression by 0.69 -fold and 0.84 -fold respectively. Several studies have suggested that spirituality and religiosity are protective factors against depression, particularly in the elderly [36-38]. In our sample, $79 \%$ of participants within the elderly age group (age of $>60$ years old) reported having a strong religious practice. Although religious practice was not a significant predictor of depression in our study when all the participants with various age groups were taken into consideration, a large proportion of elderly participants reported having a strong religious practice and the fact that increasing age reduced the odds of depression, may indicate that strong religious practice could mediate the protective effect of older age against depression in our study. A bidirectional relationship between mood disorders and diabetes has been proposed, and the occurrence of anxiety is known to increase the risk of developing depression among patients $[28,29]$. Our findings further support a bidirectional association between anxiety and depression among patients with diabetes, as reported by previous studies [9, 27]. We found that greater physical healthrelated QOL acts as a protective factor to reduce the odds of depression in diabetic patients. Similar results were found in a systematic review of 20 studies of diabetic patients across Europe and the United States [39]. Social support moderates the effect of stress on depression in which the impact of stress on depression is small in people with high social support, particularly those with high perceived spousal support $[40,41]$. This may well explain the reason why high social relationshiprelated QOL was protective against depression in our study. Regarding personality traits, unlike previous studies in the general population, our findings did not suggest a predictive effect of neuroticism on depression in patients with diabetes. This discrepancy may be explained by the moderating effect of social support on the association between neuroticism and depression. Environmental factor could be a mediating or moderating factor in the relationship between personality traits and depression [42]. Although perceived social support was not a significant predictor of depression in this study, it may reduce the effect of neuroticism on depression. Large proportion of participants reported strong social support $(80.3 \%)$ in this study. High social support may reduce the effect of neuroticism on depression as indicated by a lower odds ratio of the interaction between perceived social support and neuroticism on depression (crude OR $=1.19$ ) as compared to the odds ratio of neuroticism alone on depression (crude OR $=5.54$ ) in univariate regression analysis (Table 3 ). In the multiple regression model, neither the interaction between perceived social support and neuroticism nor neuroticism alone significantly predicted depression (Table 5).

The current study should be considered in light of its limitations. First, this study was conducted in a single tertiary healthcare referral centre. Hence, the findings may not be generalizable to the entire diabetic population in the country. Second, the cross-sectional design of the study does not allow determination of the causal relationship between the associated factors, depression and anxiety. Third, the depressive and anxiety symptoms were measured by self-reported tools rather than diagnostic interviews, which may affect the reliability of participant classification into the depressive and anxiety groups. Fourth, chronic pain is a common symptom among patients with diabetes [43], and it often coexists and interacts with anxiety and depression in these individuals [44]. However, we did not measure chronic pain as one of the predicting factors in our study, although it could be an important confounding factor which may contribute to depression and anxiety among diabetic patients.

Despite these limitations, the study had many strengths. The data obtained included a wide range of factors that could potentially be associated with depression and anxiety in diabetic patients. The study sample demonstrated diagnostic heterogeneity (patients with type 1 , type 2 , and gestational diabetes were included), representative of the Malaysian diabetic population. Our study examined the association between personality traits, quality of life, depression, and anxiety in patients with diabetes, which has previously been poorly characterized. Our study highlights a need to screen not only for psychiatric complications of diabetes, such as depression and anxiety, but also personality traits and quality of life. Hence, management of diabetes mellitus requires a multidisciplinary team that can manage both physical and mental health of patients.

\section{Conclusions}

The ADAPT-DM study reported a relatively lower prevalence of anxiety and similar prevalence of 
depression in a large and heterogeneous sample of Malaysian diabetic patients as compared to studies in other countries. Co-morbid depression and higher neuroticism increased odds of developing anxiety. Greater psychological QOL and higher conscientiousness were protective against occurrence of anxiety. Co-morbid anxiety increased the odds of developing depression, while older age, greater physical health-related QOL, and higher social relationship-related QOL were protective against depression. Our findings indicate that screening for personality traits and QOL are necessary to manage anxiety and depression for a holistic approach of diabetic treatment.

\section{Supplementary information}

Supplementary information accompanies this paper at https://doi.org/10. 1186/s12888-020-02615-y.

Additional file $\mathbf{1}$ Table S1. The mean and standard deviation for age

the WHOQOL-BREF, and the BFI scores.

\section{Abbreviations}

QOL: Quality of life; BMI: Body mass index; GAD: Generalized anxiety disorder: IQR: Interquartile range; OR: Odds ratio; $95 \%$ Cl: $95 \%$ confidence interval; ADAPT-DM: Anxiety, Depression, and Personality Traits in Diabetes Mellitus; UKMMC: Universiti Kebangsaan Malaysia Medical Centre; GAD-7: Seven-item Generalised Anxiety Disorder scale; BDI: Beck Depression Inventory-ll; BFI: Big Five Inventory; WHOQOL-BREF: World Health Organization Quality of LifeBREF; ROC curve: Receiver operating characteristic curve; AUC: Area under the receiver operating characteristic curve

\section{Acknowledgements}

We would like to thank the Centre for Research and Instrumentation, Universiti Kebangsaan Malaysia for their support in this study.

\section{Authors' contributions}

LWS-C lead the study. PJG, RLM, ESK, NHH, PA, AYZ, and NM collected the data. MFILBA prepared the original draft. HS and AR reviewed and edited the final manuscript. All authors have read and approved the final manuscript.

\section{Funding}

This research was funded by the Young Researcher Incentive Grant (Grant number: GGPM2019-024) from the Research University Fund of Universiti Kebangsaan Malaysia. The funding source had no role in the design of the study, the collection, analysis and interpretation of the data, and the writing of the manuscript.

\section{Availability of data and materials}

The datasets used and/or analysed during the current study are available from the corresponding author upon reasonable request.

\section{Ethics approval and consent to participate}

Ethics approval was obtained from Human Ethics Committee of the Faculty of Medicine, Universiti Kebangsaan Malaysia (UKM FPR.SPI 800-2/28/166/FF2019-342). All procedures performed in this study involving human participants were in accordance with the 1964 Helsinki declaration and its later amendments. Written informed consent was provided by the participants of the study.

\section{Consent for publication}

Not applicable.

\section{Competing interests}

The authors declare that they have no competing interests.

\section{Author details}

'Department of Psychiatry, Universiti Kebangsaan Malaysia Medical Center (UKMMC), 56000 Cheras, Kuala Lumpur, Malaysia. ${ }^{2}$ Faculty of Medicine, University of Toronto, 1 King's College Circle, Toronto, ON M5S 1A8, Canada. ${ }^{3}$ Faculty of Medicine, Universiti Kebangsaan Malaysia Medical Center (UKMMC), 56000 Cheras, Kuala Lumpur, Malaysia. ${ }^{4}$ Lifestyle Science Cluster, Advanced Medical and Dental Institute, Universiti Sains Malaysia,

SAINS@BERTAM, 13200 Kepala Batas, Pulau Pinang, Malaysia.

Received: 12 December 2019 Accepted: 23 April 2020

Published online: 12 May 2020

\section{References}

1. IDF diabetes atlas - 2017 Atlas. https://diabetesatlas.org/resources/2017-atlas. html. Accessed 30 Apr 2019.

2. Gojka R. WHO global report on diabetes: a summary. Int J Noncommun Dis. 2016;1 (1):3-8.

3. Kruse J, Schmitz N, Thefeld W. On the association between diabetes and mental disorders in a community sample: results from the German National Health Interview and examination survey. Diabetes Care. 2003;26(6):1841-6.

4. Sears C, Schmitz N. The relationship between diabetes and mental health conditions in an aging population. Can J Diabetes. 2016;40(1):4-5.

5. Nefs G, Hendrieckx C, Reddy P, Browne JL, Bot M, Dixon J, et al. Comorbid elevated symptoms of anxiety and depression in adults with type 1 or type 2 diabetes: results from the international diabetes MILES study. J Diabetes Complicat. 2019:33(8):523-9.

6. Khan P, Qayyum N, Malik F, Khan T, Khan M, Tahir A. Incidence of anxiety and depression among patients with type 2 diabetes and the predicting factors. Cureus. 2019;11(3):e4254.

7. Hendrieckx C, Halliday JA, Beeney $\sqcup$, Speight J. Diabetes and emotional health: a handbook for health professionals supporting adults with type 1 or type 2 diabetes. Canberra: National Diabetes Services Scheme; 2016.

8. Bădescu S, Tătaru C, Kobylinska L, Georgescu EL, Zahiu DM, Zăgrean AM, et al. The association between diabetes mellitus and depression. J Med Life. 2016;9(2):120-5.

9. Kader Maideen SF, Mohd Sidik S, Rampal L, Mukhtar F. Prevalence, associated factors and predictors of depression among adults in the community of Selangor, Malaysia. PLoS One. 2014;9(4):e95395.

10. Arambewela MH, Somasundaram NP, Jayasekara HBPR, Kumbukage MH. Prevalence of depression and associated factors among patients with type 2 diabetes attending the diabetic clinic at a tertiary care hospital in Sri Lanka: a descriptive study. Psychiatry J. 2019;2019:7468363.

11. Ahmad A, Abujbara M, Jaddou H, Younes NA, Ajlouni A. Anxiety and depression among adult patients with diabetic foot: prevalence and associated factors. J Clin Med Res. 2018;10(5):411-8.

12. Mendenhall E, Norris SA, Shidhaye R, Prabhakaran D. Depression and type 2 diabetes in low and middle income countries: a systematic review. Diabetes Res Clin Pract. 2014;103(2):276-85.

13. Salinero-Fort MA, Gómez-Campelo P, San Andrés-Rebollo FJ, CárdenasValladolid J, Abánades-Herranz JC, Carrillo de Santa Pau E, et al. Prevalence of depression in patients with type 2 diabetes mellitus in Spain (the DIADEMA Study) : results from the MADIABETES cohort. BMJ Open. 2018; 8(9):e020768.

14. Grisby AB, Anderson RJ, Freeland KE, Clouse RE, Lustman PJ. Prevalence of anxiety in adults with diabetes: a systematic review. J Psychosom Res. 2002; 53(6):1053-60

15. Spitzer RL, Kroenke K, Williams JBW, Lowe B. A brief measure for assessing generalised anxiety disorder: the GAD-7. Arch Intern Med. 2006;166(10): 1092-7.

16. Mohd Sidik S, Arroll B, Goodyear-Smith F. Validation of the GAD-7 (Malay version) among women attending a primary care clinic in Malaysia. J Prim Health Care. 2012:4(1):5-11.

17. Beck AT, Steer RA, Garbin MG. Psychometric properties of the Beck depression inventory: twenty-five years of evaluation. Clin Psychol Rev. 1988; 8(1):77-100

18. Mukhtar F, Oei TPS. Exploratory and confirmatory factor validation and psychometric properties of the Beck Depression Inventory for Malays (BDIMalay) in Malaysia. MJP Online Early. 2008;17(1). http://www. psychiatrymalaysia.org/file_dir/16510073164861a7e47a8ae.pdf. Accessed 13 Jan 2019. 
19. John OP, Donahue EM, Kentle RL. The big five inventory: versions 4a and 54. Berkeley, CA: Institute of Personality and Social Research, University of California; 1991.

20. Muhammad H, Roodenburg J, Moore DW. The adaptation of the big five inventory in measuring Malaysian youths' personality traits. Int J Adv Appl Sci. 2018:5(7):8-14.

21. Srivastava S, John OP, Gosling SD, Potter J. Development of personality in early and middle adulthood: set like plaster or persistent change? J Pers Soc Psychol. 2003;84(5):1041-53.

22. The WHOQOL Group. Development of the World Health Organization WHOQOL-BREF quality of life assessment. Psychol Med. 1998;28(3):551-8

23. Hasanah $\mathrm{Cl}$, Naing $L$, Rahman ARA. World Health Organization quality of life assessment: brief version in Bahasa Malaysia. Med J Malaysia. 2003;58(1):7988.

24. Hawthorne G, Herrman H, Murphy B. Interpreting the WHOQOL-Brèf: preliminary population norms and effect sizes. Soc Indic Res. 2006;77:37-59.

25. Oguz N. Anxiety and depression in diabetic patients. Eurasian J Med Invest. 2018;2:174-7.

26. Chaturvedi SK, Gowda SM, Ahmed HU, Alosaimi FD, Andreone N, Bobrov A, et al. More anxious than depressed: prevalence and correlates in a 15nation study of anxiety disorders in people with type 2 diabetes mellitus. Gen Psychiatr. 2019;32(4):e100076.

27. Sun N, Lou P, Shang Y, Zhang P, Wang J, Chang G. Prevalence and determinants of depressive and anxiety symptoms in adults with type 2 diabetes in China: a cross-sectional study. BMJ Open. 2016;6(8):e012540.

28. Gerontoukou E-I, Michaelidoy S, Rekleiti M, Saridi M, Souliotis K. Investigation of anxiety and depression in patients with chronic diseases. Health Psychol Res. 2015;3(2):2123.

29. Light RW, Merrill EJ, Despars JA, Gordon GH, Mutalipassi LR. Prevalence of depression and anxiety in patients with COAD. Relationship to functional capacity. Chest. 1985;87(1):35-8.

30. Baldwin DS, Evans DL, Hirschfeld RMA, Kasper S. Can we distinguish anxiety from depression? Psychopharmacol Bull. 2002;36(Suppl 2):158-65.

31. Lahey BB. Public health significance of neuroticism. Am Psychol. 2009;64(4): 241-56.

32. van der Veen DC, van Dijk SDM, Comijs HC, van Zelst WH, Schoevers RA Voshaar RCO. The importance of personality and life-events in anxious depression: from trait to state anxiety. Aging Ment Health. 2017;21(11): $1177-83$.

33. dos Santos MAB, Ceretta LB, Réus GZ, Abelaira HM, Jornada LK, Schwalm MT. Anxiety disorders are associated with quality of life impairment in patients with insulin-dependent type 2 diabetes: a case-control study. Braz J Psychiatry. 2014;36(4):298-304.

34. Martino G, Catalano A, Bellone F, Russo GT, Vicario CM, Lasco A. As time goes by: anxiety negatively affects the perceived quality of life in patients with type 2 diabetes of long duration. Front Psychol. 2019;10:1779.

35. Kotov R, Gamez W, Schmidt F, Watson D. Linking "big" personality traits to anxiety, depressive, and substance use disorders: a meta-analysis. Psychol Bull. 2010;136(5):768-821.

36. Lucchetti G, Lucchetti ALG, Peres MFP, Moreira-Almeida A, Koenig HG. Religiousness, health, and depression in older adults from a Brazilian military setting. ISRN Psychiatry. 2012;2012:940747.

37. Fiske A, Wetherell JL, Gatz M. Depression in older adults. Annu Rev Clin Psychol. 2009;5:363-89.

38. Ronneberg CR, Miller EA, Dugan E, Porell F. The protective effects of religiosity on depression: a 2-year prospective study. Gerontologist. 2016; 56(3):421-31.

39. Schram MT, Baan CA, Pouwer F. Depression and quality of life in patients with diabetes: a systematic review from the European depression in diabetes (EDID) research consortium. Curr Diabetes Rev. 2009;5(2):112-9.

40. Choi NG, Ha JH. Relationship between spouse/partner support and depressive symptoms in older adults: gender difference. Aging Ment Health. 2011;15(3):307-17.

41. Wang X, Cai L, Qian J, Peng J. Social support moderates stress effects on depression. Int J Ment Health Syst. 2014;8(1):41.

42. Klein DN, Kotov R, Bufferd SJ. Personality and depression: explanatory models and review of the evidence. Annu Rev Clin Psychol. 2011;7:269-95.

43. Krein SL, Heisler M, Piette JD, Makki F, Kerr EA. The effect of chronic pain on diabetes patients' self-management. Diabetes Care. 2005;28(1):65-70.
44. Jain $\mathrm{R}$, Jain $\mathrm{S}$, Raison $\mathrm{CL}$, Meletic V. Painful diabetic neuropathy is more than pain alone: examining the role of anxiety and depression as mediators and complicators. Curr Diab Rep. 2011;11(4):275-84.

\section{Publisher's Note}

Springer Nature remains neutral with regard to jurisdictional claims in published maps and institutional affiliations.
Ready to submit your research? Choose BMC and benefit from:

- fast, convenient online submission

- thorough peer review by experienced researchers in your field

- rapid publication on acceptance

- support for research data, including large and complex data types

- gold Open Access which fosters wider collaboration and increased citations

- maximum visibility for your research: over $100 \mathrm{M}$ website views per year

At BMC, research is always in progress.

Learn more biomedcentral.com/submissions 